\title{
Vortex critical behavior at the de-confinement phase transition
}

\author{
Kurt Langfeld* \\ Insitut für Theoretische Physik, Universität Karlsruhe \\ D-76128 Karlsruhe, Germany. \\ and \\ Insitut für Theoretische Physik, Universität Tübingen \\ Auf der Morgenstelle 14, D-72076 Tübingen, Germany.
}

(Dated: July 7, 2018)

\begin{abstract}
The de-confinement phase transition in $\mathrm{SU}(2)$ Yang-Mills theory is revisited in the vortex picture. Defining the world sheets of the confining vortices by maximal center projection, the percolation properties of the vortex lines in the hypercube consisting of the time axis and two spatial axis are studied. Using the percolation cumulant, the temperature for the percolation transition is seen to be in good agreement with the critical temperature of the thermal transition. The finite size scaling function for the cumulant is obtained. The critical index of the finite size scaling function is consistent with the index of the 3D Ising model.
\end{abstract}

PACS numbers: 11.15.Ha, 12.38.Aw, 12.38.Gc

Understanding de-confinement at finite temperatures is one of the major challenges of QCD, the theory of strong interactions. Large scale collision experiments (RHIC) are currently operating in order to detect new states of matter at high temperatures [1, 2]. The general believe is that the confinement of quarks is induced by the gluonic state, and that the mechanism can be anticipated within the pure $S U(N)$ gauge theory. Using dimensional reduction in combination with lattice simulations of the emerging effective 3D theory, it was recently shown [7] that the non-perturbative effects of $S U(N)$ gauge theory are strong for temperatures which are accessible in collider experiments. The universality class of the transition is of particular importance because the corresponding degrees of freedom are weakly interacting close to the transition, thus dictating the dynamics of the theory.

Any theory which purports certain degrees of freedom of being relevant for confinement should also be able to explain de-confinement at finite temperatures. An example for such degrees of freedom are chromomagnetic monopoles (a pedagogical review to the subject can be found in [3]). It was empirically observed that monopole currents, residing in the spatial hypercube, form a network which ceases percolation in the de-confined phase [4, 5]. However, it seems likely that the monopoles are strongly coupled even close to the de-confinement transition. Indeed, arguments have been put forward that a (weakly interacting) Coulomb gas of chromo-magnetic monopoles is inconsistent with the phenomenology of confinement [6].

Below, I will address two scenarios of the deconfinement phase transition at finite temperatures. One of these exploits the realization of the global $Z(N)$ cen-

*Electronic address: kurt.langfeld@uni-tuebingen.de ter symmetry of the corresponding $S U(N)$ Yang-Mills theories. The spontaneous breaking of this symmetry at high temperatures is signaled by a non-vanishing expectation value of the Polyakov line. In the case of the SU(2) gauge theory, the projection of the Polyakov line to the center elements has revealed that the effective theory of the corresponding 3D Ising model is short ranged at the transition [8, 9, 10]. The thermal transition corresponds to a percolation transition of the site-bond clusters of the emerging 3D Ising model. More recently, it was observed [1] that also the ratio of the Polyakov line critical amplitudes are in agreement with that of the 3D Ising model. These findings confirm that the transition of the $\mathrm{SU}(2)$ gauge theory belongs to the universality class of the $Z_{2}$ Ising model in three dimensions.

A second scenario emerged with the advent of the socalled maximum center gauge (MCG) [6, 12, 13, 14]: a tight relation between quark confinement and the vortex structure of the pure gluonic vacuum was established at a quantitative level for $S U(2)$ and $S U(3)$ [15, 16] gauge theory: projecting $S U(2)$ lattice configurations onto those of a $Z_{2}$ gauge theory, the confining capabilities of the static quark anti-quark potential at large distances is retained. On the other hand, removing the vortex structure from the lattice configurations "by hand", the static potential looses its linear rise and resembles a Coulomb potential. The key observation is that the vortices which emerge from the above effective $Z_{2}$ gauge theory [14 are sensible degrees of freedom of continuum Yang-Mills theory: extrapolating to the continuum limit of vanishing lattice spacing, the planar vortex density [14, 17] as well as the binary vortex interactions [18] are finite in units of the fundamental energy scale (e.g. string tension). These observations indicate that the MCG vortices are the degrees of freedom relevant for quark confinement. Recently, it was shown that the vortices might also play a role for other low energy observables: the removal of the vortex structure leads to a restoration of chiral symmetry [19] 

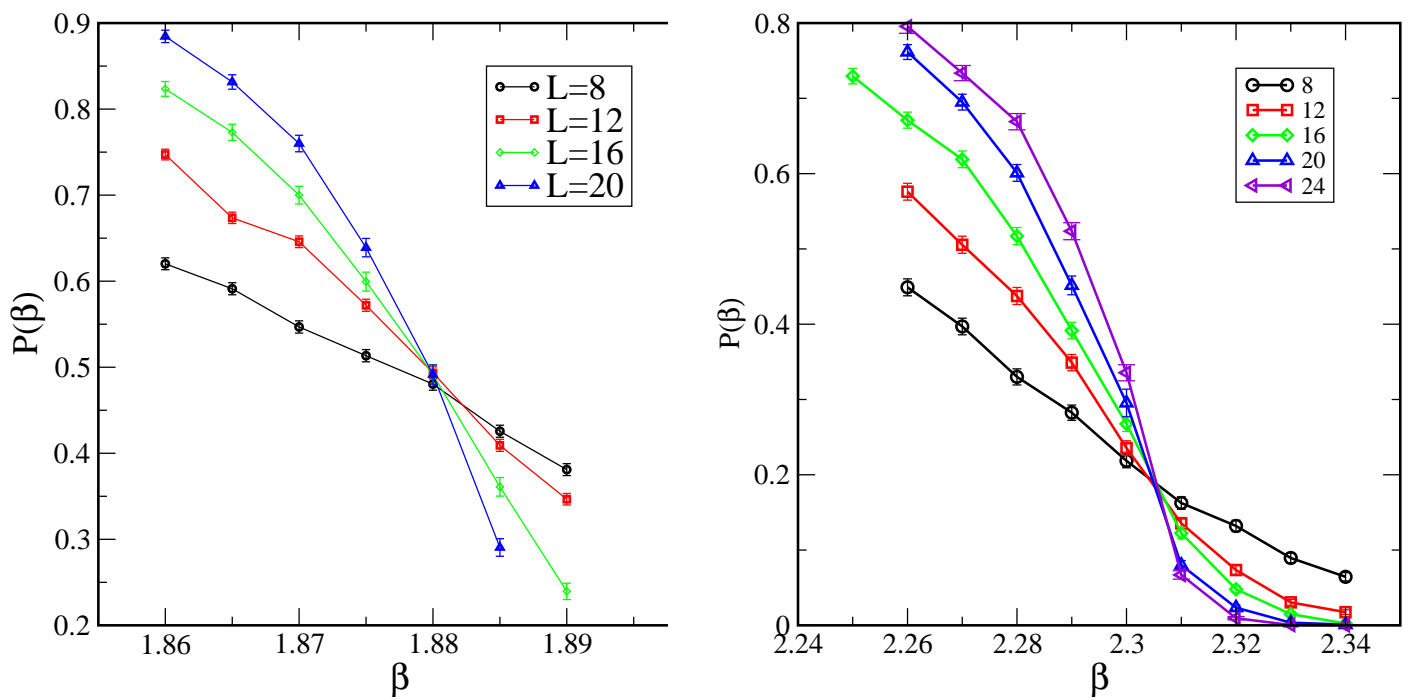

FIG. 1: The percolation cumulant $P(\beta)$ for several lattice sizes $L$ for $N_{t}=2$ (left) and $N_{t}=4$ (right).

and to a strong reduction of the infrared strength of certain Yang-Mills Greenfunctions [20, 21].

The question whether one can grasp the essence of the de-confinement phase transition at finite temperatures within the vortex picture was firstly investigated in 22, 23. One finds that the effective $Z_{2}$ theory which arises from the $S U(2)$ lattice gauge configurations after center projection [14] reproduces the right order of magnitude of the de-confinement temperature $T_{c}$. In order to detect the change of the vortex structure when $T_{C}$ is approached, the vortex world lines of a hypercube consisting of the time and two spatial directions were studied. While the vortices percolate at small temperatures, the vortices align parallel to the time axis direction for $T>T_{c}$. The de-confinement phase transition appears as vortex de-percolation transition.

A comparison of both scenarios of the de-confinement phase transition which have been discussed above suggests a certain duality: (i) the Polyakov line gives rise to a Ising model defined in the spatial hypercube; sitebond clusters start percolating in the high temperature phase; (ii) closed vortex loops emerge from MCG projection in the hypercube spanned by the time- and two spatial directions; the vortex clusters cease to percolate in the de-confinement phase. In order to establish a duality between both pictures, one must verify that the critical behavior of the vortex percolation transition corresponds to the 3D Ising universality class.

In this letter, a detailed analysis of the center vortex loops in the time-like hypercube is performed at finite temperatures. Using the percolation cumulant, a precise determination of the critical de-percolation temperature $T_{p}$ is obtained. I find that $T_{p}$ nicely agrees with the thermal transition temperature $T_{c}$. Finally, the finite size scaling function of the percolation cumulant will show that the finite size scaling is consistent with that of the 3D Ising model.

The links $U_{\mu}(x)$ representing the $S U(2)$ gauge field configurations are generated with the standard Wilson action. The Maximal Center Gauge is installed by maximizing the functional

$$
\sum_{x, \mu}\left[\operatorname{tr} U_{\mu}^{\Omega}(x)\right]^{2} \stackrel{\Omega}{\rightarrow} \max
$$

where $U_{\mu}^{\Omega}(X)=\Omega(x) U_{\mu}(x) \Omega^{\dagger}(x+\mu)$ are the gauged links. Finding the global maximum of (10) is a highly nontrivial task and is subject to Gribov ambiguities. One observes that bulk properties of the vortices are little affected by the choice of algorithm (see e.g. 20]). Here, I will not further address the Gribov problem, but will adopt a practical point of view: I will employ the procedure described in 14 because it yields good scaling properties of the vortex matter. One must, however, keep in mind that it is not clear to which extent the choice of algorithm affects the results presented here. Center projection is then performed by

$$
S U(2) \rightarrow Z_{2}: U_{\mu}^{\Omega}(x) \rightarrow Z_{\mu}(x)=\operatorname{sign} \operatorname{tr} U_{\mu}^{\Omega}(x) .
$$

Defining

$$
v(p):=\prod_{l \in p} Z_{l}, \quad l=\{x, \mu\}
$$

one says that a vortex pierces an elementary plaquette $p$ if $v(p)=-1$. Hence, vortices exists on the dual lattice. In view of the $Z_{2}$ Biancchi identity,

$$
\prod_{p \in c} v(p)=1
$$



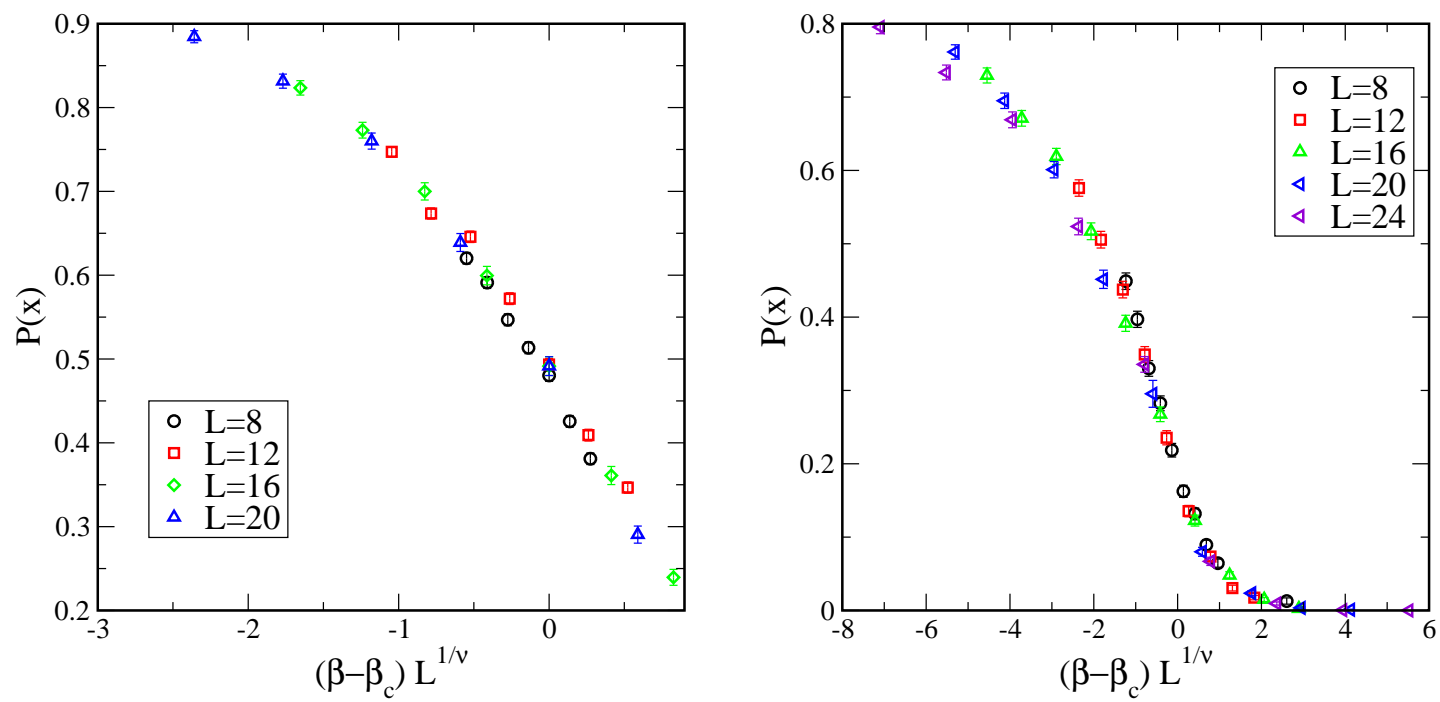

FIG. 2: The percolation cumulant $P(x)$ for several lattice sizes $L$ for $N_{t}=2$ (left) and $N_{t}=4$ (right) as function of the scaling variable $x$ with $\nu=0.6294$ as input.

where $c$ denotes an elementary cube, vortices form closed world sheets in 4 dimensions and appear as closed loops in a particular 3D hypercube. In the following, we will consider the time-like hypercube spanned by the timeand two spatial directions, and we will investigate the properties of the closed vortex loops within this hypercube. A vortex cluster is defined as the vortex material which is connected by a single vortex world line. The time-like hypercube contains $L^{2} \times N_{t}$ lattice points. The temperature $T=1 / N_{t} a(\beta)$ is adjusted by varying $\beta$. Below, results for $N_{t}=2$ and $N_{t}=4$ are presented.

For an investigation of the vortex percolation within the time-like hypercube, one determines the minimal box which completely contains a given vortex cluster. If this box is as big as the time-like hypercube, the particular cluster is said to percolate. In order to study the vortex critical behavior at the critical temperature, one defines the so-called percolation cumulant []$] P(\beta)$, which is given by the average number of percolating vortex cluster divided by the number of configurations. Analogous to the Binder cumulant, the percolation cumulant is independent of the extent of the lattice $L$ at the critical point $\beta_{c}$. This implies that the curves $P(\beta)$ corresponding to different lattice sizes cross at the critical point. My numerical results are shown in figure 1 I find

$$
\beta_{c}=1.880(3) \quad\left(N_{t}=2\right), \quad \beta_{c}=2.305(3) \quad\left(N_{t}=4\right) .
$$

These values are in reasonable agreement with those quoted for the phase transition signaled by the percolation of the Polyakov line [8], i.e.,

$$
\begin{array}{lll}
\beta_{c}^{\text {pol }} & =1.8734(2) & \left(N_{t}=2\right), \\
\beta_{c}^{\text {pol }} & =2.29895(10) & \left(N_{t}=4\right) .
\end{array}
$$

As long as finite size scaling holds, the percolation cumulant should be a universal function of

$$
x:=\left(\beta-\beta_{c}\right) L^{1 / \nu},
$$

where $\nu$ is characteristic for the universality class of the phase transition. Figure 2 shows the vortex percolation cumulant as function the scaling variable $x$ for $N_{t}=2$ and $N_{t}=4$. The numerical results are consistent with the critical index $\nu=0.6294$ of the the 3D Ising model. It is remarkable that the finite size scaling works for a wide range of the scaling variable $x$.

In conclusions, the picture of the de-confinement phase transition at finite temperatures as a vortex depercolation transition is refined: the finite size scaling function of the vortex percolation cumulant is obtained. The critical index $\nu$ is seen to be consistent with the index of the 3D Ising model. Hence, the center vortex picture of the phase transition is put on equal footing as the percolation picture of site-bond clusters defined by the Polyakov line [8]-[10]. Since the definition of the Polyakov line breaks covariance, the latter picture is necessarily restricted to temperatures of the order of the deconfinement temperature. By contrast, the center vortex clusters are consistently defined with the symmetries at all temperatures, and provide a picture dual to the Polyakov line picture at $T \approx T_{c}$. However, one must keep in mind that the Gribov problem renders an unambiguous definition of the vortex cluster cumbersome at the present stage of research.

Acknowledgments: I thank M. Quandt for helpful comments on the manuscript. 
[1] U. W. Heinz, The quark-gluon plasma at RHIC, arXiv:nucl-th/0212004

[2] D. Kharzeev, Important experimental observables at RHIC, arXiv:nucl-th/0211083

[3] M. N. Chernodub and M. I. Polikarpov, arXiv:hep-th/9710205

[4] V. G. Bornyakov, V. K. Mitrjushkin and M. MullerPreussker, Phys. Lett. B 284, 99 (1992).

[5] G. Damm and W. Kerler, Phys. Lett. B 397, 216 (1997) arXiv:hep-lat/9702012.

[6] J. Greensite, arXiv:hep-lat/0301023

[7] K. Kajantie, M. Laine, K. Rummukainen and Y. Schroder, Phys. Rev. Lett. 86, 10 (2001) arXiv:hep-ph/0007109.

[8] S. Fortunato, F. Karsch, P. Petreczky and H. Satz, Phys. Lett. B 502, 321 (2001) arXiv:hep-lat/0011084.

[9] S. Fortunato and H. Satz, Nucl. Phys. A 681, 466 (2001) arXiv:hep-lat/0007012.

[10] H. Satz, Comput. Phys. Commun. 147, 46 (2002) arXiv:hep-lat/0110013.

[11] J. Engels and T. Scheideler, Nucl. Phys. B 539, 557 (1999) arXiv:hep-lat/9808057.

[12] L. Del Debbio, M. Faber, J. Greensite and S. Olejnik, Phys. Rev. D 55, 2298 (1997) arXiv:hep-lat/9610005.

[13] L. Del Debbio, M. Faber, J. Greensite and S. Olejnik, arXiv:hep-lat/9708023
[14] L. Del Debbio, M. Faber, J. Giedt, J. Greensite and S. Olejnik, Phys. Rev. D 58, 094501 (1998) arXiv:hep-lat/9801027.

[15] M. Faber, J. Greensite and S. Olejnik, Phys. Lett. B 474, 177 (2000) arXiv:hep-lat/9911006.

[16] J. D. Stack, W. W. Tucker and R. J. Wensley, Nucl. Phys. B 639, 203 (2002).

[17] K. Langfeld, H. Reinhardt and O. Tennert, Phys. Lett. B 419, 317 (1998) arXiv:hep-lat/9710068.

[18] M. Engelhardt, K. Langfeld, H. Reinhardt and O. Tennert, Phys. Lett. B 431, 141 (1998) arXiv:hep-lat/9801030.

[19] P. de Forcrand and M. D'Elia, Phys. Rev. Lett. 82, 4582 (1999) arXiv:hep-lat/9901020.

[20] K. Langfeld, H. Reinhardt and J. Gattnar, Nucl. Phys. B 621, 131 (2002) arXiv:hep-ph/0107141.

[21] K. Langfeld, J. C. Bloch, J. Gattnar, H. Reinhardt, A. Cucchieri and T. Mendes, arXiv:hep-th/0209173

[22] K. Langfeld, O. Tennert, M. Engelhardt and H. Reinhardt, Phys. Lett. B 452, 301 (1999) arXiv:hep-lat/9805002.

[23] M. Engelhardt, K. Langfeld, H. Reinhardt and O. Tennert, Phys. Rev. D 61, 054504 (2000) arXiv:hep-lat/9904004. 